\title{
XPC inhibition rescues cisplatin resistance via the Akt/mTOR signaling pathway in A549/DDP lung adenocarcinoma cells
}

\author{
XUE TENG $^{1 *}$, XIAO-FAN FAN ${ }^{1 *}$, QI LI $^{2}$, SHUANG LIU $^{1}$, DONG-YUAN WU ${ }^{1}$, \\ SHU-YA WANG ${ }^{1}$, YUANQI SHI ${ }^{3}$ and MEI DONG ${ }^{1}$
}

Departments of ${ }^{1}$ Pharmacy and ${ }^{2}$ Biotherapy Center, Harbin Medical University Cancer Hospital; ${ }^{3}$ Department of Cardiology, The First Affiliated Hospital of Harbin Medical University, Harbin, Heilongjiang 150086, P.R. China

Received July 20, 2018; Accepted January 3, 2019

DOI: $10.3892 / o r .2019 .6959$

\begin{abstract}
Xeroderma pigmentosum, complementation group $\mathrm{C}$ (XPC) is an accessory recognition gene involved in the nucleotide excision repair (NER) pathway, which is activated during the initial DNA damage recognition stage. It participates in the regulation of DNA damage-induced proliferation and apoptosis. Emerging evidence demonstrates that upregulation of XPC increases the resistance of several tumor cell types to cytotoxic drugs. In addition, it can predict poor patient outcome for non-small cell lung cancer (NSCLC). However, the mechanisms linking upregulation of XPC and drug resistance in lung cancer are still unclear. In the present study, we aimed to confirm whether XPC was involved in the reversal of the cisplatin (DDP) resistance in drug-resistant A549/DDP lung adenocarcinoma cells. RT-PCR and western blot assays were used to examine XPC mRNA and protein expression levels. Cell viability was assessed by CCK-8 assay. The knockdown of XPC was achieved in A549/DDP cells using si-RNA, whereas cell proliferation and apoptosis were assessed by wound healing assay and flow cytometric
\end{abstract}

Correspondence to: Dr Yuanqi Shi, Department of Cardiology, The First Affiliated Hospital of Harbin Medical University, 23 Postal Street, Harbin, Heilongjiang 150086, P.R. China

E-mail:kean1943@126.com

Dr Mei Dong, Department of Pharmacy, Harbin Medical University Cancer Hospital, 150 Haping Road, Harbin, Heilongjiang 150086, P.R. China

E-mail: mdhappy2006@hotmail.com

*Contributed equally

Abbreviations: NSCLC, non-small cell lung cancer; DDP, cisplatin; NER, nucleotide excision repair; XPC, xeroderma pigmentosum complementation group C; MMP1, matrix metalloproteinase-1; CCK-8, Cell Counting Kit-8; ERCC1, excision repair cross-complementing 1; XPA, xeroderma pigmentosum, complementation group A; XPF, xeroderma pigmentosum, complementation group F; A549/DDP, cisplatin-resistant A549 cells

Key words: XPC, DDP, NSCLC, resistance analysis, respectively. The median inhibitory concentration $\left(\mathrm{IC}_{50}\right)$ value of DDP was assessed by CCK-8 assay. Western blot assays were conducted for the examination of caspase-9/3, Bax and Bcl-2 protein levels, whereas the activation of the $\mathrm{PI} 3 \mathrm{~K} / \mathrm{Akt} / \mathrm{mTOR}$ signaling pathway was investigated in XPC-knockdown cells. High expression of XPC was noted in A549/DDP cells compared with that in A549 cells, which was associated with DDP resistance. XPC silencing significantly inhibited A549/DDP cell proliferation and increased the induction of apoptosis. In addition, XPC knockdown decreased the expression levels of the Akt/mTOR signaling proteins and the expression of their downstream mediator. The data of the present study revealed that XPC inhibition rescued DDP resistance in lung adenocarcinoma cells, which was dependent on the Akt/mTOR signaling pathway. Collectively, XPC may be considered a new strategy for curing DDP-resistant lung cancer and may improve the efficacy of conventional chemotherapy.

\section{Introduction}

Lung cancer is the leading cause of cancer mortality worldwide and its incidence is constantly increasing (1). Non-small cell lung cancer (NSCLC) is the main pathological type of lung cancer and its incidence is $>85 \%$ in patients with lung cancer. Platinum-based dual drug regimens are the first-line treatment for NSCLC $(1,2)$. Cisplatin (DDP) is a classic platinum compound used in lung cancer therapy, which binds to DNA strands and interferes with DNA replication causing cell cycle arrest in the S phase (3). However, the increased ability of DNA repair frequently affects the therapeutic efficacy of DDP. Thus, it is indispensable to explore the mechanism of drug resistance and provide alternative means to improve the antitumor efficacy of DDP. Recent studies have proposed numerous mechanisms that could explain DDP chemoresistance, including decreased drug accumulation in cells, enhanced detoxification ability, enhanced DNA damage repair function of the cells and inhibition or inactivation of the apoptotic process $(4,5)$. However, DDP resistance in the aforementioned cases is associated with the increased ability of DNA repair and nucleotide excision repair (NER) that are involved in the removal of intra-strand DNA-cross-links caused by DDP (6). 
Xeroderma pigmentosum, complementation group C (XPC) is a nuclear excision repair gene which mainly participates in the identification of DNA damage (7). Recent studies have identified that XPC is aberrantly expressed in several human types of cancer, such as lung, breast, gastric and ovarian cancer (8-11). The positive correlation of the expression of XPC and the outcome of various types of cancer indicated that XPC was closely associated with the incidence of cancer and its progression. This in turn affected the response rate and the overall survival rate of the affected patients. Emerging evidence has suggested that XPC affects early stages of lung carcinogenesis (12). It is important to note that the expression deficiency or downregulation of XPC affects DNA repair capacity and the sensitivity of the cells to platinum-based drug chemotherapy (13). Thus, several studies have shown that XPC expression deficiency can activate matrix metalloproteinase-1 (MMP1) and $\mathrm{p} 53$ transcription in order to promote induction of apoptosis (14). The present study revealed that XPC may play major roles in the progression of lung cancer via its sensitivity to standard chemotherapy (14). However, the exact mechanism underlying the upregulation of XPC expression levels and drug resistance in lung cancer remains unclear.

In the present study, we aimed to investigate the role of XPC on the resistance of A549/DDP lung adenocarcinoma cells with regard to the efficacy of DDP. The expression levels of the XPC protein and mRNA were determined in A549 and A549/DDP cells. The proliferative, migratory and apoptotic activities of A549/DDP cells were examined in comparison with XPC expression using gene silencing approaches. The signaling pathways that mediated the induction of XPC expression were investigated. The findings revealed that XPC silencing significantly inhibited A549/DDP cell proliferation and increased the induction of apoptosis. XPC further regulated the major proteins involved in the PI3K/Akt/mTOR signaling pathway and their downstream mediators in vitro.

\section{Materials and methods}

Reagents and antibodies. DDP was obtained from SigmaAldrich; Merck (Shanghai, China). XPC-siRNA (si-XPC) and the negative control (siNC) were purchased from Shanghai GeneChem Co., Ltd. (Shanghai, China). The FITC Annexin V Apoptosis Detection kit 1 (BD Biosciences, Franklin Lakes, NJ, USA) was used for detecting cell apoptosis by flow cytometry. The anti-Akt (dilution 1:1,000; cat. no. 4691S), anti-p-Akt (dilution 1:1,000; cat. no. 4060S), anti-caspase-3 (dilution 1:1,000; cat. no. 9662S), anti-cleaved-caspase-3 (dilution 1:1,000; cat. no. 9661S) and anti-caspase-9 (dilution 1:1,000; cat. no. 9502P) antibodies were purchased from Cell Signaling Technology, Inc. (Danvers, MA, USA); and the anti-p-mTOR (dilution 1:1,000; cat. no. 13152-1) antibody was purchased from Signalway Antibody LLC (College Park, MD, USA). The anti-PI3K (dilution 1:1,000; cat. no. A0982) and anti-mTOR (dilution 1:1,000; cat. no. A11928) antibodies were purchased from ABclonal Technology (Wuhan, China), and the anti-XPC (dilution 1:2,000; cat. no. ab203693) antibody was purchased from Abcam (Cambridge, UK). The anti-Bax (dilution 1:1,000; cat. no. 50599-1-AP) and anti-Bcl-2 (dilution 1:1,000; cat. no. 12789-1-AP) antibodies were purchased from ProteinTech Group, Inc. (Rosemont,
IL, USA). The actin (dilution 1:1,000; cat. no. TA-09) and GAPDH (dilution 1:1,000; cat. no. TA-08) antibodies were obtained from OriGene Technologies, Inc. (Beijing, China). The secondary antibody horseradish peroxidase conjugated goat anti-rabbit IgG (dilution 1:5,000; cat. no. ZB-2306), also was purchased from OriGene Technologies, Inc.

Cell culture and transfection. A549 and A549/DDP human lung carcinoma cells were obtained from the Chinese Academy of Sciences (Shanghai, China). Both cell lines were cultured in RPMI-1640 medium (HyClone; GE Healthcare Life Sciences, Logan, UT, USA) supplemented with $10 \%$ fetal bovine serum (FBS; HyClone; GE Healthcare Life Sciences), $100 \mathrm{U} / \mathrm{ml}$ penicillin and $100 \mathrm{U} / \mathrm{ml}$ streptomycin (Gibco; Thermo Fisher Scientific, Inc., Waltham, MA, USA) in a humidified atmosphere of $5 \% \mathrm{CO}_{2}$ at $37^{\circ} \mathrm{C}$. In addition, A549/DDP cells were cultured in medium containing $2 \mathrm{mg} / 1 \mathrm{DDP}$ in order to retain the drug resistant phenotype. The cells were divided into 3 groups as follows: The blank group (without transfection), the group transfected with the small interfering RNA targeting the $X P C$ (si-XPC) group and the negative control (si-NC) group. The target sequence of si-XPC was: CTCTGACCTGTTACA AGTA. A total of $2 \times 10^{5}$ A549/DDP cells were added into a 6 -well plate. The cells were transfected at $70 \%$ confluence with si-XPC and/or si-NC using Lipofectamine ${ }^{\mathrm{TM}} 2000$ (Invitrogen; Thermo Fisher Scientific, Inc.) according to the manufacturer's instructions. The transfection reagent was mixed with si-RNA or si-NC in a ratio of $2 \mu \mathrm{l}: 1 \mu \mathrm{g}$. We determined the transfection efficiency using western blotting. Following $24 \mathrm{~h}$ of transfection, each group of cells that were in the logarithmic growth phase was collected for the next experiment.

Cell Counting Kit-8 assay. Cell Counting Kit-8 (CCK-8) assay (Dojindo Molecular Technologies, Inc., Rockville, MA, USA) was used to detect cell viability following different treatments. Briefly, A549 and A549/DDP cells were transfected with si-XPC and si-NC and were incubated in a 96-well plate (3,000 cells/well) overnight. Following DDP treatment at the indicated doses $(0,0.5,1,2,4,8,16$ and $32 \mathrm{mg} / \mathrm{l})$ for $24 \mathrm{~h}$, $10 \mu 1$ of CCK-8 solution was added to each well and the plates were incubated for $1 \mathrm{~h}$. The optical density (OD) of each well was detected at $450 \mathrm{~nm}$ using a microplate reader (Bio-Rad Laboratories, Inc., Hercules, CA, USA) according to the manufacturer's instructions. The half inhibitory concentration $\left(\mathrm{IC}_{50}\right)$ of the cells was determined by CCK- 8 assay. The half-maximal inhibitory concentration $\left(\mathrm{IC}_{50}\right)$ of the drugs was calculated on GraphPad Prism using the $\log$ (inhibitor) vs. response-variable slope (4 parameters) equation under the non-linear regression dialogue.

Wound healing assay. Following transfection of A549/DDP cells, the cells were allowed to grow for $24 \mathrm{~h}$. The monolayer was scratched with a $200-\mu 1$ pipette tip. The attached cells were washed 3 times with phosphate-buffered saline (PBS) in order to remove floating cells and debris. Subsequently, serum-free medium was added. Moreover, DDP was added in the petri dishes as described above. The cells were continuously incubated for $48 \mathrm{~h}$. The wounds were visualized every $24 \mathrm{~h}$, and the lines that were aligned with the wounds of each group were photographed in each experiment under an 
inverted microscope (TS100; Nikon Corp., Tokyo, Japan). In each well, at least 8 regions of each condition was captured randomly at a magnification of x100. The images were analyzed using ImageJ v2.1.4.7 software (National Institutes of Health, Bethesda, MD, USA).

Flow cytometry. The rate of apoptosis was analyzed by flow cytometry using an Annexin V-FITC/PI kit (BD Biosciences, Franklin Lakes, NJ, USA). A549/DDP cells were seeded in a 6-well plate and incubated overnight. Following transfection, the cells were incubated with DDP in growth media for $24 \mathrm{~h}$. The cells were collected and resuspended in $500 \mu \mathrm{l}$ of $1 \mathrm{X}$ binding buffer. Subsequently, staining was conducted as described by the manufacturer's protocol.

Quantitative real-time PCR. Total RNA was extracted with TRIzol reagent (Axygen, Inc.; Corning Inc., Corning, NY, USA). The cDNA was generated with a cDNA Synthesis kit (Toyobo Life Science, Osaka, Japan). qRT-PCR was performed using a SYBR-Green assay kit (Roche Diagnostics, Basel, Switzerland) on an Applied Biosystems thermal cycler (Applied Biosystems; Thermo Fisher Scientific, Inc.). The qPCR reactions were run on the ABI 7500 real-time PCR system using the following conditions: $95^{\circ} \mathrm{C}$ for $30 \mathrm{sec}$, followed by 35 cycles at $62^{\circ} \mathrm{C}$ for $30 \mathrm{sec}$ and $72^{\circ} \mathrm{C}$ for $30 \mathrm{sec}$, final extension at $72^{\circ} \mathrm{C}$ for $5 \mathrm{~min}$. The relative mRNA expression levels of GAPDH were calculated and quantified with the $2^{-\triangle A C q}$ method (15). GAPDH was used as an internal control. The primers for XPC and GAPDH were as follows: 5'-GAC AAGCAGGAGAAGGCAAC-3' and 5'-GGTTCGGAATCC TCATCAGA-3' for the XPC sense and reverse primers, respectively; and 5'-TGGACCTGACCTGCCGTCTA-3' and 5'-AGGAGTGGGTGTCGCTGTTG-3' for the GAPDH sense and reverse primers, respectively.

Western blot assay. The expression levels of various proteins were detected by western blot analysis. The total protein in the cells was collected by RIPA lysis buffer (Beijing Solarbio Science \& Technology Co., Ltd., Beijing, China). The protein concentration was determined using a BCA protein concentration assay kit (Beyotime Institute of Biotechnology, Shanghai, China), and protein products $(8-12 \mu \mathrm{g} / \mu \mathrm{l} ; 40-50 \mu \mathrm{g}$ total) were separated by $10 \%$ sodium dodecyl sulfate-polyacrylamide gel electrophoresis (SDS-PAGE) and then transferred onto polyvinylidene fluoride (PVDF) membranes (EMD Millipore, Bedford, MA, USA). Subsequently, the membranes were blocked with BSA for $1 \mathrm{~h}$ at room temperature and incubated with primary antibodies overnight at $4^{\circ} \mathrm{C}$. Following washing with PBST (Beijing Solarbio Science \& Technology Co., Ltd.), the PVDF membranes were subsequently incubated with the secondary antibodies for $1 \mathrm{~h}$. Following additional washing with PBST to remove the secondary antibodies, the protein signals were detected by the ChemiDoc XRS gel documentation system (Bio-Rad Laboratories, Inc., Hercules, CA, USA) using ECL Western Blotting Substrate (Beijing Solarbio Science and Technology Co., Ltd.). The results were scanned and quantified using the ImageJ software v2.1.4.7.

Statistical analysis. Each experiment was conducted at least 3 times and the results were presented as the mean values \pm standard deviation (SD). One-way analysis of variance (ANOVA) was carried out to compare the differences among multiple groups, and the Bonferroni test was performed followed ANOVA. In addition, the independent samples t-test was performed to compare the differences between two groups. Statistical analysis was performed using the GraphPad Prism 5 statistical software (GraphPad Software, Inc., San Diego, CA, USA). A P-value of $<0.05$ was considered to indicate a statistically significant difference.

\section{Results}

Upregulation of XPC in A549/DDP cells. The cytotoxic effects of different concentrations of DDP $(0,0.5,1,2,4$, 8,16 and $32 \mathrm{mg} / \mathrm{l}$ ) were examined in A549/DDP and A549 cells. A CCK-8 assay was used to assess cell viability. The results revealed that the growth inhibitory rate was increased following the increase in the concentration of DDP, while the inhibitory effect of DDP on A549 cells was significantly higher than that noted in A549/DDP cells (Fig. 1A). Furthermore, we calculated the $\mathrm{IC}_{50}$ values of A549 and A549/DDP cells using a CCK- 8 assay, and the results indicated that it was $6.43 \pm 0.89 \mathrm{mg} / \mathrm{l}$ in A549 cells and $14.6 \pm 2.87 \mathrm{mg} / 1$ in A549/DDP cells. A549/DDP cells were more resistant to DDP treatment in vitro than A549 cells and $10 \mathrm{mg} / \mathrm{l}$ of DDP was selected as the optimal concentration in the follow-up experiments (Fig. 1B).

Western blot assays indicated that the expression levels of XPC were significantly increased in A549/DDP cells compared with those noted in A549 cells $(\mathrm{P}<0.05)$ (Fig. 1C). Moreover, to determine whether upregulation of XPC expression was a result of increased transcription, qRT-PCR was performed to analyze the mRNA levels of $X P C$. The results demonstrated that the mRNA levels of XPC were also upregulated (Fig. 1D). DDP treatment induced an increase in XPC protein levels in A549/DDP cells (Fig. 1E). These results indicated that the expression levels of XPC were upregulated in A549/DDP cells compared with those in A549 cells.

Efficient silencing of XPC expression in A549/DDP cells using siRNA. To further explore the role of XPC in the resistance of A549/DDP cells to chemotherapy, XPC knockdown in A549/DDP cells was performed with siRNA. Based on previous studies, the most effective siRNA (siRNA-178) was selected from the 3 knockdown sequences (siRNA-177/178/179) for the following experiments. A549/DDP cells were transfected with si-XPC or si-NC. Western blotting detection demonstrated that the expression levels of XPC were significantly downregulated compared with those noted in the negative control group (Fig. 2A). Furthermore, $X P C$ mRNA levels were also inhibited following si-XPC transfection (Fig. 2B). The effects of XPC knockdown on the sensitivity of the resistant cells to DDP were subsequently investigated. A CCK-8 assay was employed to examine the cell viability of A549/DDP cells. The results revealed that the inhibitory potencies of DDP were higher in the si-XPC transfected group than those in the other 2 groups (Fig. 2C). In addition, the $\mathrm{IC}_{50}$ values of DDP in the 3 groups were calculated and as shown in Fig. 2D, no significant differences were noted in the $\mathrm{IC}_{50}$ values of DDP between the control $(14.6 \pm 2.87 \%)$ and the negative control groups $(14.81 \pm 2.36 \%)$. 


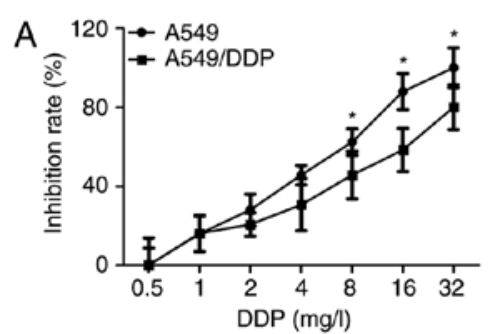

D

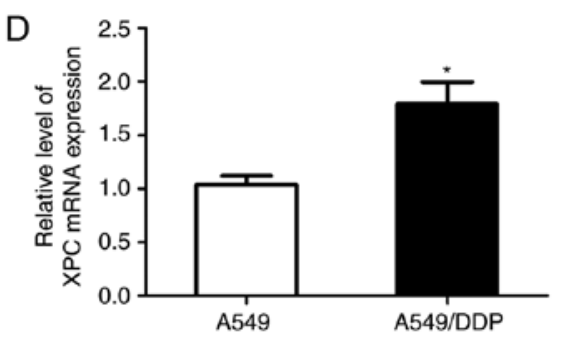

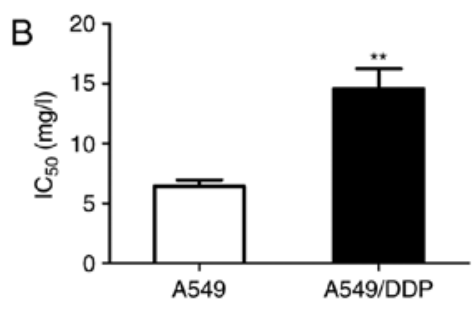

E
C
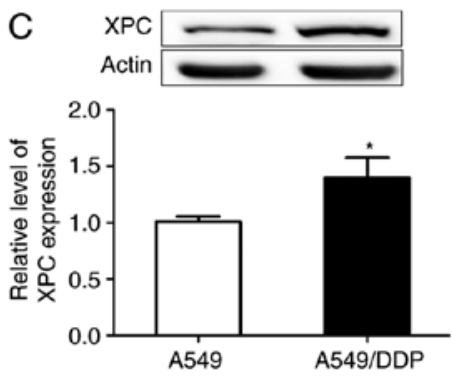
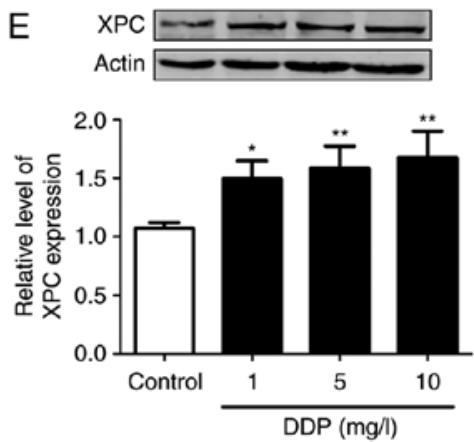

Figure 1. $\mathrm{IC}_{50}$ and XPC expression in A549 and A549/DDP cells. (A) Following treatment of the cells with different concentrations of DDP, a CCK-8 assay was used to detect cell viability. (B) The $\mathrm{IC}_{50}$ values of the A549/DDP and A549 cells exposed to DDP. (C) Western blot assays demonstrated the increased protein expression of XPC in A549/DDP cells. The bands were quantified with ImageJ software. ${ }^{*} \mathrm{P}<0.05,{ }^{* *} \mathrm{P}<0.01$ vs. A549 cells. (D) qRT-PCR was used to detect the increased mRNA levels of XPC in A549/DDP cells. ${ }^{*} \mathrm{P}<0.05$ vs. A549 cells. (E) Expression of XPC protein in A549/DDP cells following a dose-dependent treatment of DDP. ${ }^{*} \mathrm{P}<0.05,{ }^{* *} \mathrm{P}<0.01$ vs. the control cells. The data were expressed as the mean $\pm \mathrm{SD} ; \mathrm{n}=3$. XPC, xeroderma pigmentosum, complementation group C; CCK-8, Cell Counting Kit-8; DDP, cisplatin.

A
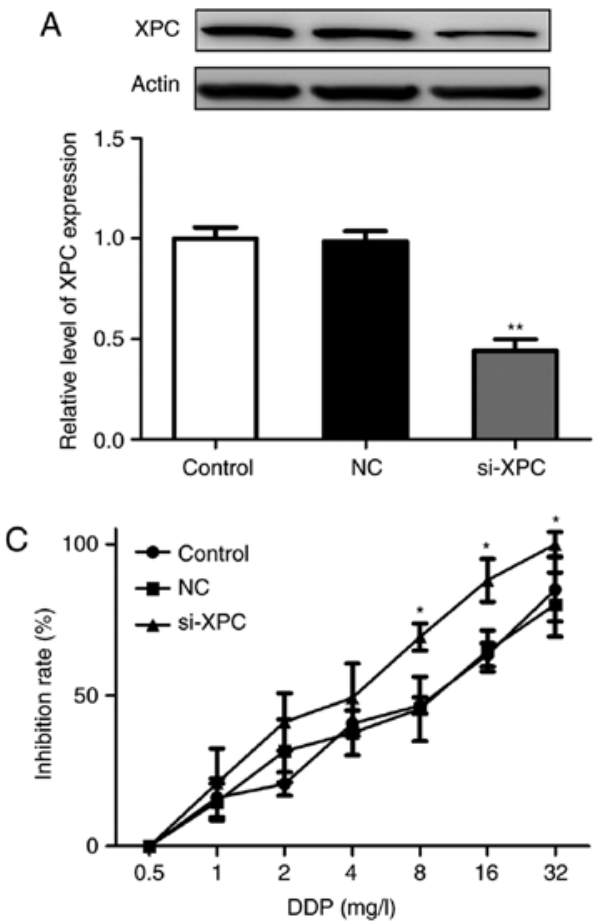
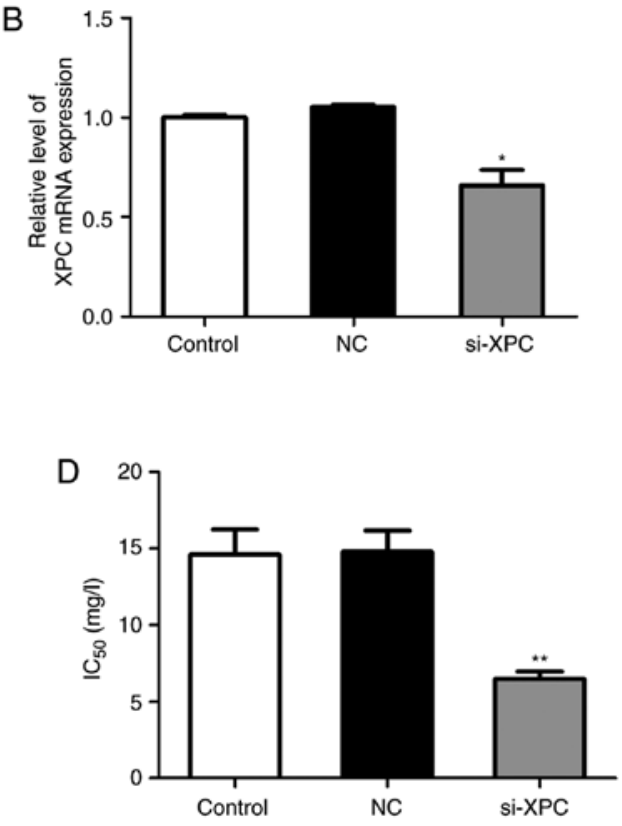

Figure 2. Effects of XPC silencing by siRNA on the proliferation of A549/DDP cells. (A) Western blot assays were used to detect the expression levels of the XPC protein following transfection. (B) qRT-PCR analysis of XPC was performed. (C) The effects of DDP treatment on the proliferation of A549/DDP cells. (D) The $\mathrm{IC}_{50}$ values of the cells from the control, siRNA negative control, and si-XPC transfected groups. The data were presented as the mean $\pm \mathrm{SD}$; $\mathrm{n}=3$ ${ }^{*} \mathrm{P}<0.05,{ }^{* *} \mathrm{P}<0.01$ vs. the control or NC groups. si-XPC, small interfering XPC gene; NC, negative control; XPC, xeroderma pigmentosum, complementation group C; DDP, cisplatin.

In contrast to these observations, the $\mathrm{IC}_{50}$ value of $\mathrm{DDP}$ was significantly reduced in the si-XPC transfected group
$(6.5 \pm 0.82)$ compared with that noted in the other 2 groups $(\mathrm{P}<0.05)$. These results indicated that A549/DDP cells were 
A

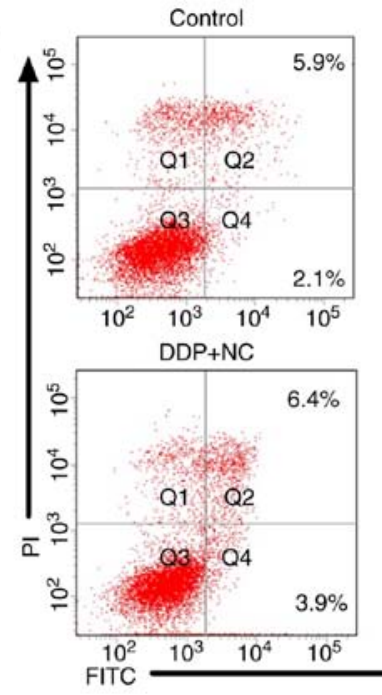

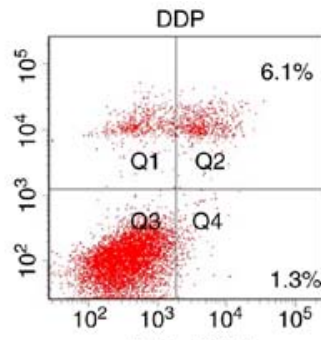
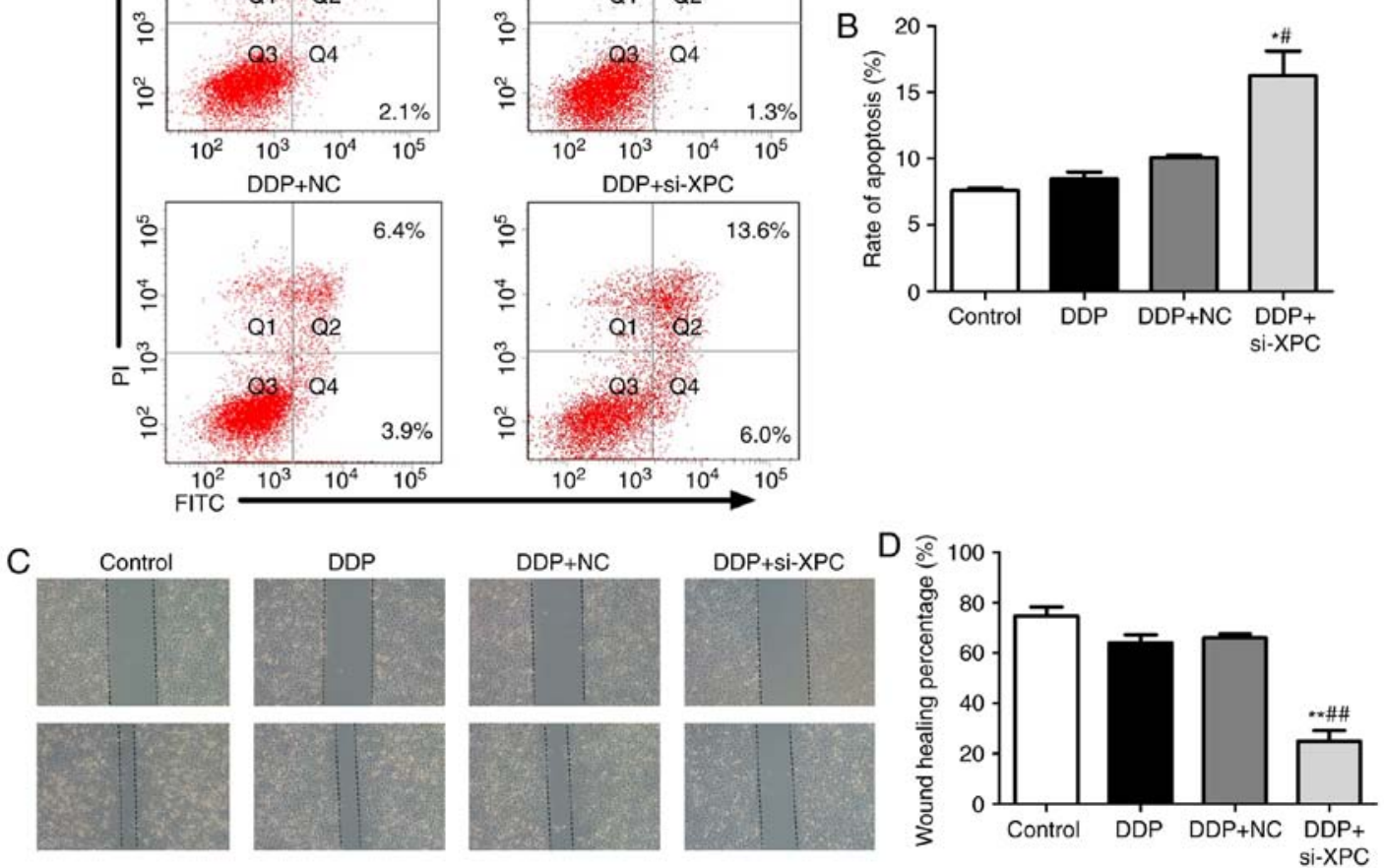

Figure 3. XPC silencing promotes apoptosis and reduces proliferation and migration. (A) Representative flow cytometric analysis of cell apoptosis using Annexin V-FITC/PI double staining following siRNA transfection and addition of DDP for $24 \mathrm{~h}$. The right lower quadrant in the dot plot represents the early stage of the apoptotic cells. (B) Quantitative analysis of the percentage of apoptotic cells in the early stage. (C) Wound healing assay was performed for motility of A549/DDP cells following transfection and addition of DDP. The images were captured at 0 and $48 \mathrm{~h}$ following the scratch (magnification, $\mathrm{x} 100$ ). (D) Quantitative analysis of the percentage of wound healing. The data were presented as the mean $\pm \mathrm{SD} ; \mathrm{n}=3$. ${ }^{*} \mathrm{P}<0.05,{ }^{* *} \mathrm{P}<0.01 \mathrm{vs}$. the control group, ${ }^{\#} \mathrm{P}<0.05$, ${ }^{\#} \mathrm{P}<0.01$ vs. the DDP group. XPC, xeroderma pigmentosum, complementation group C; DDP, cisplatin.

more sensitive to DDP treatment following efficient silencing of XPC.

Silencing of XPC in A549/DDP cells by si-XPC reduces proliferation and migration and promotes apoptosis. The effect of XPC knockdown on the induction of apoptosis using Annexin V-FITC/PI double staining was explored. As revealed in Fig. $3 \mathrm{~A}$ and $\mathrm{B}$, the apoptotic rate of the si-XPC group was increased to $19.6 \%$ in the A549/DDP cells, indicating that XPC knockdown induced apoptosis in A549/DDP cells. A CCK- 8 assay was used to detect the proliferative ability of A549/DDP cells. The results revealed that the proliferation of A549/DDP cells was significantly reduced following transfection with si-XPC (Fig. 2C). Furthermore, a wound healing assay was used to detect the migratory ability of A549/DDP cells. The results demonstrated that the migratory distance noted in A549/DDP cells was significantly reduced following transfection with si-XPC (Fig. 3C and D). Subsequently, the expression levels of the apoptosis-related proteins, including caspase-3, caspase-9, Bax, Bcl-2 and active caspase- 3 were also determined by western blot assays. XPC knockdown increased the expression levels of active caspase-3/caspase-3 and Bax/Bcl-2, while it decreased the expression levels of caspase-9 in A549/DDP cells that were treated with DDP for $24 \mathrm{~h}$ (Fig. 4). These results indicated that XPC knockdown inhibited cell proliferation and survival.
Silencing of XPC in A549/DDP cells activates the PI3K/Akt/ mTOR pathway. In order to investigate whether the $\mathrm{PI} 3 \mathrm{~K} / \mathrm{Akt} / \mathrm{mTOR}$ pathway is involved in the XPC-mediated cell apoptosis of A549/DDP cells, the expression levels of apoptosis-related proteins were detected. Initially, the expression levels of the phosphorylated form of the Akt protein (Ser473) were significantly increased in drug-resistant cells, while no significant change was noted in the expression levels of the total Akt protein (Fig. 5A). Subsequently, DDP-resistant cells were treated with PI3K inhibitors and western blotting was performed in order to detect the expression levels of XPC. The results revealed that XPC expression in the treatment group was significantly lower than that in the control group (Fig. 5B). This indicated a potential interaction between $\mathrm{p}-\mathrm{Akt}$ and XPC.

XPC silencing using siRNA did not affect the protein levels of PI3K (Fig. 5C). Silencing of XPC caused a significant decrease in the expression levels of p-Akt and p-mTOR proteins in A549/DDP cells (Fig. 5D and E). However, transfection of si-XPC did not result in a significant change in the protein levels of Akt and mTOR. This transfection caused a decrease in the levels of the phosphorylated (activated) form of mTOR (Ser2448), a downstream target of PI3K/Akt, which has been revealed to promote cell growth. The results of the present study indicated the potential impact of XPC on the Akt/mTOR signaling pathway. Furthermore, silencing of XPC increased the levels of active caspase-3 protein following DDP 

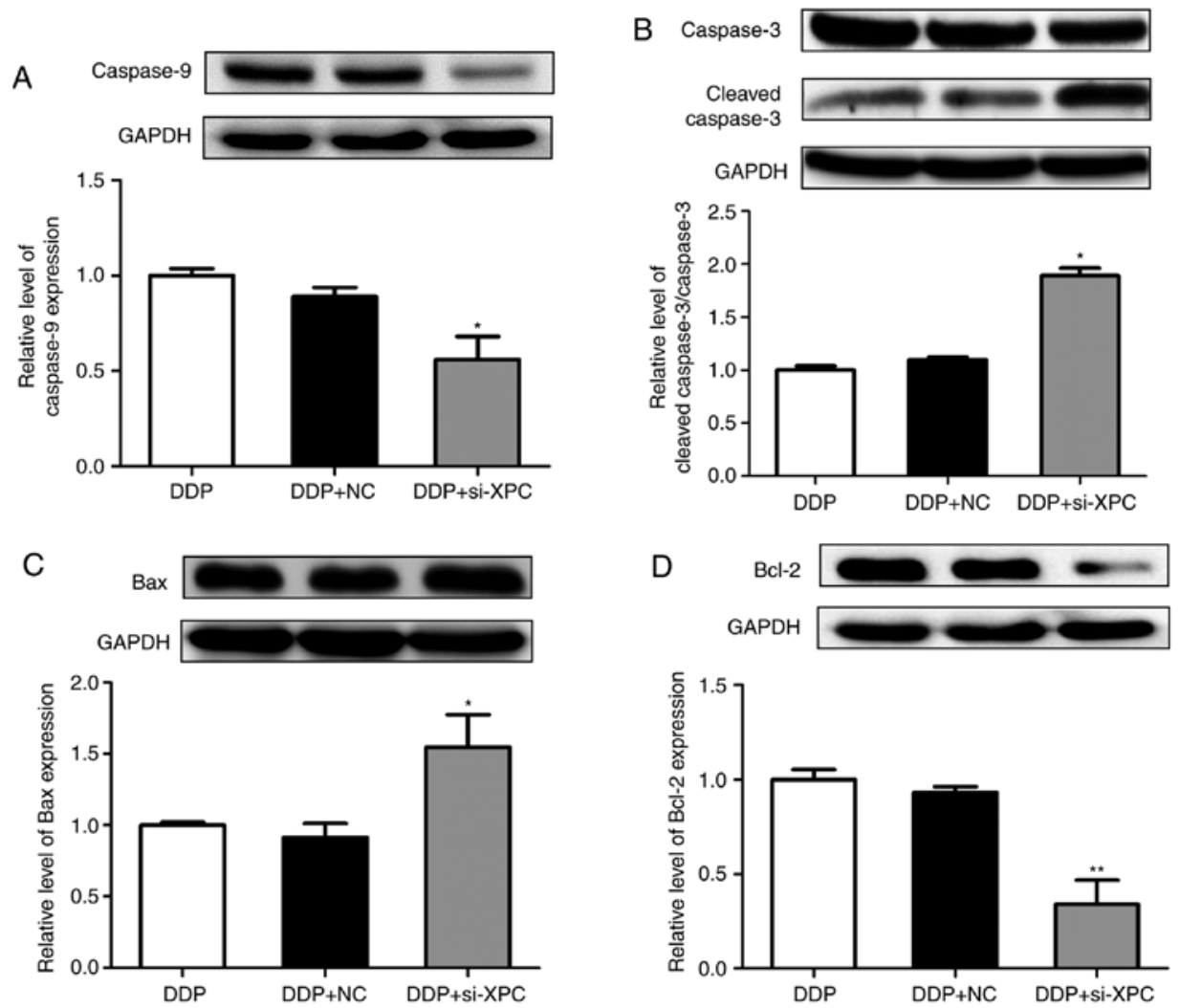

Figure 4. The expression of the apoptotic proteins following XPC silencing. (A-D) Western blot analysis of caspase-3/9, active caspase-3, Bax and Bcl-2 proteins was performed. GAPDH was used as an internal control. The data were presented as the mean $\pm \mathrm{SD} ; \mathrm{n}=3$. ${ }^{*} \mathrm{P}<0.05,{ }^{* *} \mathrm{P}<0.01$. XPC, xeroderma pigmentosum, complementation group $\mathrm{C}$.
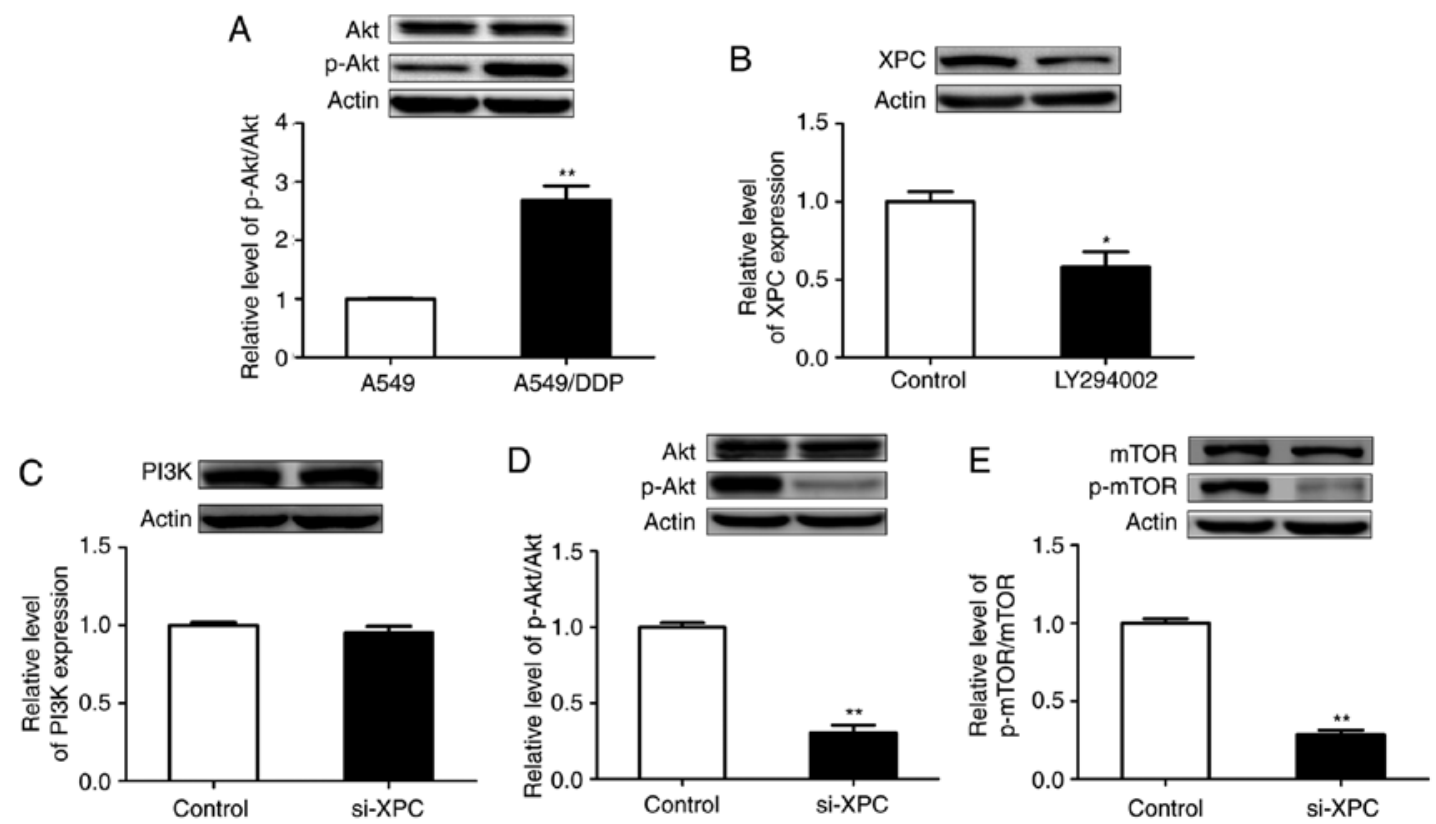

Figure 5. The effects of XPC silencing on the phosphoinositide 3-kinase/Akt/mTOR pathway. (A) The expression levels of the Akt and p-Akt proteins were examined by western blotting in A549 and A549/DDP cells. (B) Western blot analysis of XPC in A549/DDP cells was performed in the presence of a PI3K inhibitor. (C) The expression levels of the PI3K protein in the control and si-XPC groups was examined by western blot assays. (D and E) The expressions levels of Akt and p-Akt proteins in the control and si-XPC groups were examined by western blot assays. The protein expression levels of mTOR and p-mTOR were also detected by western blot analysis. $\beta$-actin was used as an internal control. The data were presented as the mean $\pm \mathrm{SD} ; \mathrm{n}=3$. ${ }^{*} \mathrm{P}<0.05$, ${ }^{* *} \mathrm{P}<0.01$. $\mathrm{XPC}$, xeroderma pigmentosum, complementation group C; DDP, cisplatin; p, phosphorylated; mTOR, mammalian target of rapamycin.

treatment (Fig. 4B). Collectively, the data indicated the effects of XPC on the Akt/mTOR signaling pathway and confirmed that XPC plays a vital role in the regulation of apoptosis in A549/DDP cells. 


\section{Discussion}

The resistance of cancer cells to conventional cytotoxic drugs ultimately leads to chemotherapy failure and increased patient mortality. DDP, a non-specific cell cycle agent, has been used as a primary treatment against various malignancies, notably those of lung, gastric, esophageal and ovarian origin. The DNA repair capacity can maintain the genome integrity and chromosomal stability. Therefore, the increased ability of DNA repair can lead to DDP resistance. The NER is a key process required for DNA repair (6). Various studies have recently revealed that the defective expression of NER-related genes is associated with tumor progression and DDP resistance. The results indicated that the reduced levels of excision repair cross-complementing 1 (ERCC1), xeroderma pigmentosum, complementation group A (XPA) or xeroderma pigmentosum, complementation group $\mathrm{F}$ (XPF) were associated with the development and prognosis of lung carcinoma (16). Furthermore, in vitro studies have linked high ERCC1 expression with platinum resistance in several types of cancer, while the detection of $E R C C 1$ has been used as a biomarker for individualized treatment in lung cancer patients (17-19). A previous study examined the silencing of various NER genes in HeLa cells and demonstrated that the suppression of XPC expression could lead to an increased sensitivity for etoposide treatment combined with DDP (20). Furthermore, upregulation of XPC has been revealed in lung adenocarcinoma tissue samples of DDP insensitive patients (21). The increased expression levels of XPC caused by the stimulation of the cells with DDP indicate a potential biological mechanism of genomic integrity leading to DDP resistance.

The serine/threonine kinase Akt is involved in the progression of various types of cancer, whereas the Akt-mediated signaling pathway plays an anti-apoptotic role by phosphorylating target proteins in various downstream pathways $(22,23)$. The PI3K/Akt/mTOR is an important intracellular signaling pathway that regulates the cell cycle during cell dormancy, proliferation, cancer progression and necrosis. However, a previous study has revealed that XPC expression levels were related to the inactivation of the Akt pathway (24). Recently, several studies have also revealed that Akt inhibition reduces cell survival and improves DDP resistance $(25,26)$. Cheng et al reported that the levels of PI3K and pAkt in lung cancer cells were significantly increased compared with those in normal cells and that the application of PI $3 \mathrm{~K}$ inhibitors could promote lung cancer cell apoptosis (27). Thus, we hypothesized that XPC could alleviate the resistance of DDP through the Akt signaling pathway in lung cancer cells.

In the present study, we propose a novel mechanism that can reverse the effects of XPC on DDP resistance in lung cancer cells. Initially, the results indicated that XPC mRNA and XPC protein levels were markedly increased in the resistant cells (A549/DDP cells) compared with those noted in the parent cells (A549 cells) $(\mathrm{P}<0.05)$. In addition, the present study examined the regulation of A549/DDP cell growth by detecting the induction of apoptosis and the expression of the PI3K/Akt signaling pathway proteins. The downregulation of XPC expression following transfection with si-RNA increased the sensitivity to DDP and reduced the expression of the p-Akt and p-mTOR proteins. Concomitantly, the proliferative ability and the high $\mathrm{IC}_{50}$ value were significantly reduced in A549/DDP cells following XPC downregulation compared with those noted in the negative control samples.

It has been confirmed that caspase- 3 and $\mathrm{Bax} / \mathrm{Bcl}-2$ are involved in the regulation of apoptosis. The present study demonstrated that the ratios of cleaved caspase- $3 /$ caspase -3 and $\mathrm{Bax} / \mathrm{Bcl}-2$ were significantly increased following knockdown of XPC expression in resistant cells (A549/DDP cells), while the $\mathrm{IC}_{50}$ value and p-Akt expression levels of these cells were reduced. Our results indicated that suppression of XPC expression could significantly increase the induction of apoptosis in chemotherapy-resistant cells. Therefore, we hypothesized that XPC plays an essential role in the regulation of caspase-3, and in determining the $\mathrm{Bax} / \mathrm{Bcl}-2$ ratio in lung cancer cells. These effects can reverse the resistance of A549/DDP cells to DDP by accelerating the induction of apoptosis and by inhibiting cancer cell proliferation. The resistance of lung cancer cells to chemotherapeutic drugs was associated with high XPC expression.

In conclusion, our results demonstrated that XPC expression was linked with DDP resistance in lung cancer cells and that the reversal of the resistance was mainly mediated by the Akt/mTOR signaling pathway. The present study provides significant findings in the potential clinical applications of XPC as a prognostic marker for DDP resistance of NSCLC. Moreover, this application can aid the efficacy of chemotherapy by providing personalized treatment for each patient based on his pharmacogenomic profile.

\section{Acknowledgements}

Not applicable.

\section{Funding}

The present study was supported by the Heilongjiang Provincial Health and Family Planning Commission Scientific Research Subject (no. 2017-120), the Harbin Medical University Cancer Hospital Haiyan Fund Youth funding project (no. JJQN2014-04), the National Natural Science Foundation of China (81700303) and the Fundamental Research Funds for the Provincial Universities (2017LCZX17).

\section{Availability of data and materials}

All data used in the present study were included in this manuscript.

\section{Authors' contributions}

YS and MD designed the study. XT and XFF wrote the manuscript. XT, XFF, QL and SL performed the experiments including cell culture, cell transfection and cell apoptosis assays. SL, DYW and SYW participated in the western blot assays. DYW and SYW participated in the real-time RT-PCR assays. YS, XT and QL conducted the statistical analysis. YS and MD revised the manuscript. All authors read and approved the manuscript and agreed to be accountable for all aspects of the research in ensuring that the accuracy or integrity of any part of the work were appropriately investigated and resolved. 


\section{Ethics approval and consent to participate}

Not applicable.

\section{Patient consent for publication}

Not applicable.

\section{Competing interests}

The authors declare that they have no competing interests.

\section{References}

1. Siegel RL, Miller KD and Jemal A: Cancer statistics, 2015. CA Cancer J Clin 65: 5-29, 2015.

2. NSCLC Meta-Analyses Collaborative Group: Chemotherapy in addition to supportive care improves survival in advanced non-small-cell lung cancer: A systematic review and meta-analysis of individual patient data from 16 randomized controlled trials. J Clin Oncol 26: 4617-4625, 2008.

3. Siddik ZH: Cisplatin: Mode of cytotoxic action and molecular basis of resistance. Oncogene 22: 7265-7279, 2003.

4. Amable L: Cisplatin resistance and opportunities for precision medicine. Pharmacol Res 106: 27-36, 2016.

5. Milane L, Ganesh S, Shah S, Duan ZF and Amiji M: Multi-modal strategies for overcoming tumor drug resistance: Hypoxia, the Warburg effect, stem cells, and multifunctional nanotechnology. J Control Release 155: 237-247, 2011.

6. De Silva IU, McHugh PJ, Clingen PH and Hartley JA: Defining the roles of nucleotide excision repair and recombination in the repair of DNA interstrand cross-links in mammalian cells. Mol Cell Biol 20: 7980-7990, 2000.

7. Shell SM, Hawkins EK, Tsai MS, Hlaing AS, Rizzo CJ and Chazin WJ: Xeroderma pigmentosum complementation group C protein (XPC) serves as a general sensor of damaged DNA. DNA Repair (Amst) 12: 947-953, 2013.

8. Fautrel A, Andrieux L, Musso O, Boudjema K, Guillouzo A and Langouët S: Overexpression of the two nucleotide excision repair genes ERCC1 and XPC in human hepatocellular carcinoma. J Hepatol 43: 288-293, 2005.

9. Yang LC, Hsiao YP, Lu CT, Huang CH, Chao WR, Lin YT, Su HA, Chang SL and Chung JG: Xeroderma pigmentosum complementation group C Protein (XPC) expression in basal cell carcinoma. In Vivo 29: 35-38, 2015.

10. Zhang Y, Yu JJ, Tian Y, Li ZZ, Zhang CY, Zhang SF, Cao LQ, Zhang Y, Qian CY, Zhang W, et al: eIF3a improve cisplatin sensitivity in ovarian cancer by regulating XPC and p $27^{\mathrm{Kipl}}$ translation. Oncotarget 6: 25441-25451, 2015.

11. Zhang Y, Cao J, Meng Y, Qu C, Shen F and Xu L: Overexpression of xeroderma pigmentosum group $\mathrm{C}$ decreases the chemotherapeutic sensitivity of colorectal carcinoma cells to cisplatin. Oncol Lett 15: 6336-6344, 2018.

12. Wang C, Nie H, Li Y, Liu G, Wang X, Xing S, Zhang L, Chen X, Chen Y and Li Y: The study of the relation of DNA repair pathway genes SNPs and the sensitivity to radiotherapy and chemotherapy of NSCLC. Sci Rep 6: 26526, 2016.

13. Hollander MC, Philburn RT, Patterson AD, Velasco-Miguel S, Friedberg EC, Linnoila RI and Fornace AJ Jr: Deletion of XPC leads to lung tumors in mice and is associated with early events in human lung carcinogenesis. Proc Natl Acad Sci USA 102: 13200-13205, 2005.
14. Wu YH, Wu TC, Liao JW, Yeh KT, Chen CY and Lee H: p53 dysfunction by xeroderma pigmentosum group $\mathrm{C}$ defects enhance lung adenocarcinoma metastasis via increased MMP1 expression. Cancer Res 70: 10422-10432, 2010.

15. Livak KJ and Schmittgen TD: Analysis of relative gene expression data using real-time quantitative PCR and the 2(-Delta Delta C(T)) method. Methods 25: 402-408, 2001.

16. Jordheim LP, Crosperrial E, Matera EL, Bouledrak K and Dumontet C: Expression of domains for protein-protein interaction of nucleotide excision repair proteins modifies cancer cell sensitivity to platinum derivatives and genomic stability. Clin Exp Pharmacol Physiol 41: 817-824, 2014.

17. Du P, Zhang X, Liu H and Chen L: Lentivirus-mediated RNAi silencing targeting ERCC1 reverses cisplatin resistance in cisplatin-resistant ovarian carcinoma cell line. DNA Cell Biol 34: 497-502, 2015.

18. Wang Z, Liang X, Cheng Z, Xu Y, Yin P, Zhu H, Li Q, Qian X and Liu J: Induction of apoptosis and suppression of ERCC1 expression by the potent amonafide analogue 8-c in human colorectal carcinoma cells. Anticancer Drugs 24: 355-365, 2013.

19. Wang W, Zhang L, Liu L, Zheng Y, Zhang Y, Yang S, Shi R and Wang S: Chemosensitizing effect of shRNA-mediated ERCC1 silencing on a Xuanwei lung adenocarcinoma cell line and its clinical significance. Oncol Rep 37: 1989-1997, 2017.

20. Despras E, Pfeiffer P, Salles B, Calsou P, Kuhfittig-Kulle S, Angulo JF and Biard DS: Long-term XPC silencing reduces DNA double-strand break repair. Cancer Res 67: 2526-2534, 2007.

21. Lai TC, Chow KC, Fang HY, Cho HC, Chen CY, Lin TY, Chiang IP and Ho SP: Expression of xeroderma pigmentosum complementation group $\mathrm{C}$ protein predicts cisplatin resistance in lung adenocarcinoma patients. Oncol Rep 25: 1243-1251, 2011.

22. Zhang K, Wang X and Wang H: Effect and mechanism of Src tyrosine kinase inhibitor sunitinib on the drug-resistance reversal of human A549/DDP cisplatin-resistant lung cancer cell line. Mol Med Rep 10: 2065-2072, 2014.

23. Zhou D, Liu W, Liang S, Sun B, Liu A, Cui Z, Han X and Yuan L: Apoptin-derived peptide reverses cisplatin resistance in gastric cancer through the PI3K-AKT signaling pathway. Cancer Med 7: 1369-1383, 2018

24. Rezvani HR, Kim AL, Rossignol R, Ali N, Daly M, Mahfouf W, Bellance N, Taïeb A, de Verneuil H, Mazurier F and Bickers DR: XPC silencing in normal human keratinocytes triggers metabolic alterations that drive the formation of squamous cell carcinomas. J Clin Invest 121: 195-211, 2011.

25. Jiang W, Cheng Y, Zhao N, Li L, Shi Y, Zong A and Wang F: Sulfated polysaccharide of Sepiella Maindroni ink inhibits the migration, invasion and matrix metalloproteinase- 2 expression through suppressing EGFR-mediated p38/MAPK and $\mathrm{PI} 3 \mathrm{~K} / \mathrm{Akt} / \mathrm{mTOR}$ signaling pathways in SKOV-3 cells. Int J Biol Macromol 107: 349-362, 2018.

26. Zhao M, Xu P, Liu Z, Zhen Y, Chen Y, Liu Y, Fu Q, Deng X, Liang Z, Li Y, et al: Dual roles of miR-374a by modulated c-Jun respectively targets CCND1-inducing PI3K/AKT signal and PTEN-suppressing Wnt/ $/$-catenin signaling in non-small-cell lung cancer. Cell Death Dis 9: 78, 2018.

27. Cheng H, Zou Y, Ross JS, Wang K, Liu X, Halmos B, Ali SM, Liu H, Verma A, Montagna C, et al: RICTOR amplification defines a novel subset of lung cancer patients who may benefit from treatment with mTOR $1 / 2$ inhibitors. Cancer Discov 5: 1262-1270, 2015 